\title{
Malfunction of the Extracorporeal Circulation System: Case Report
}

\author{
Carlos Eduardo David de Almeida ${ }^{1}$, Antônio Roberto Carraretto, TSA ${ }^{2}$, Erick Freitas Curi, TSA ${ }^{3}$, \\ Louisie Marcelle da Silva Almeida Marques ${ }^{4}$, Roberta Eleni Monteiro Abatti 5
}

Summary: Almeida CED, Carraretto AR, Curi EF, Marques LMSA, Abatti REM - Malfunction of the Extracorporeal Circulation System: Case Report.

Background and objectives: The introduction of extracorporeal circulation in clinical practice was decisive for the development of modern cardiovascular surgery. Addition of new procedures and equipment, however, brings inherent risks and complications. The objective of this report is to describe a malfunction of the oxygenation system and emphasize the importance of the interaction among the medical team members to prevent errors and complications.

Case Report: During valve replacement and IVC correction surgery, we observed a darker shade of red in the blood on the exit of the oxygenator. Laboratory tests demonstrated severe acidosis and hypoxemia. The entire system was evaluated, but the cause of the malfunction was not found. Measures to reduce damage were successfully instituted. After the surgery, the whole system underwent technical evaluation.

Conclusions: Interaction among the medical team members, early diagnosis, and immediate intervention were fundamental for a favorable outcome.

Keywords: Intraoperative Complications; Extracorporeal Circulation; Accident Prevention.

\section{INTRODUCTION}

Advancements in cardiac surgeries were only possible thanks to the development of the apparatus responsible for the bypass of blood flow from the heart, allowing correction of lesions under its direct view. Previously, open heart surgery was only possible with the use of hypothermia and cardiac arrest, with important limitations of the surgical time.

The use of extracorporeal circulation (ECC) as a support method in cardiovascular surgeries is relatively recent. The

Received from Universidade Federal do Espírito Santo (UFES); Hospital Universitário Cassiano Antônio Moraes HUCAM-UFES, Brazil.

1. TEA MEC/SBA from Universidade de São Paulo (USP); Assistant Physician at Serviço de Anestesiologia do Hospital Universitário Cassiano Antônio Moraes (HUCAM) of Universidade Federal do Espírito Santo (UFES)

2. PhD in Anesthesiology, Universidade Estadual de São Paulo (UNESP); Professor of the Departamento de Clínica Cirúrgica da UFES; Responsible for the CET Integrado HUCAMHospital Geral (HAFPES)

3. President of SAES; Co-responsible for the CET Integrado HUCAM-HAFPES; Assistant Physician at Serviço de Anestesiologia do HUCAM-UFES

4. TEA MEC/SBA, UFES; Assistant Physician at Serviço de Anestesiologia do HUCAMUFES

5. Anesthesiology Resident at CET Integrado HUCAM-HAFPES

Submitted on November 4, 2010.

Approved on February 21, 2011.

Correspondence to

Dr. Carlos Eduardo David de Almeida

Av. Cesar Helal, 1181, apt 1903

Praia do Suá

29052230 - Vitória, ES, Brazil

E-mail: cedalmeida@terra.com.br concept of artificial circulation was idealized in the nineteenth century by Le Gallois; however, its successful clinical application only occurred in the twentieth century. In 1953, John Gibbon corrected an interatrial communication in a young 18-year old female using an artificial heart-lung system ${ }^{1}$.

In addition to the foreseen physiologic changes with the use of ECC, complications from equipment malfunction have been reported ${ }^{2-4}$. Failures of the EEC equipment may be related to circulation pumps (electrical or mechanical failures) and with oxygenators and circuits (cracks, disconnections, embolism, hemolysis, obstruction of blood or gas flow, defect or leakage of the heat-exchanger, and defect on the gas blender). The use of halogenated anesthetic agents through the oxygenator gas line can cause fractures in components of the ECC system ${ }^{2}$. Thus, all professionals of the surgical team, including the anesthesiologist, should know how the system works and its possible intercurrences.

The objective of this report was to present a complication due to malfunctioning of the ECC system.

\section{CASE REPORT}

This is a 49-year old male patient, $63 \mathrm{~kg}$, with aortic regurgitation and interventricular communication (IVC), who was admitted for biological valve replacement and surgical correction of IVC. Monitoring included continuous electrocardiogram (ECG), pulse oximetry $\left(\mathrm{SpO}_{2}\right)$, invasive blood pressure (IBP), 
capnography and capnometry $\left(\mathrm{EtCO}_{2}\right)$, gas analyzer, esophageal temperature ( $\mathrm{C}$ ), and central venous pressure (CVP).

Patient was medicated with oral midazolam $15 \mathrm{mg}, 30$ minutes before surgery. Intravenous induction was performed with fentanyl $\left(7.5 \mu \mathrm{g} . \mathrm{kg}^{-1}\right)$, propofol $\left(1 \mathrm{mg} \cdot \mathrm{kg}^{-1}\right)$, and pancuronium (0.06 mg. $\left.\mathrm{kg}^{-1}\right)$. Anesthesia was maintained with isoflurane (0.5-1 MAC) and continuous infusion of sufentanil, as needed.

The procedure was performed without intercurrences until the onset of circulatory support. After respiratory arrest, the anesthesiologist observed a darker shade of red in the blood on the exit of the membrane oxygenator. Arterial blood gases showed severe acidosis and hypoxemia with the following values: $\mathrm{pH} 7.07, \mathrm{PaCO}_{2} 67.3 \mathrm{mmHg}, \mathrm{PaO}_{2} 108 \mathrm{mmHg}, \mathrm{BE}$ -9.6 mmol.L-1, $\mathrm{HCO}_{3} 15.8 \mathrm{mmol}^{-1}{ }^{-1}, \mathrm{SatO}_{2} 33 \%$. The entire system was immediately checked, but no apparent irregularities were observed. It was not possible to remove the patient from assisted circulation as cardiotomy had been already performed. Hypothermia was then initiated with active cooling, administration of sodium thiopental, increasing of oxygen flow through the oxygenator, and reestablishement of pulmonary ventilation. A temporary shunt was established between the systemic and pulmonary circulation.

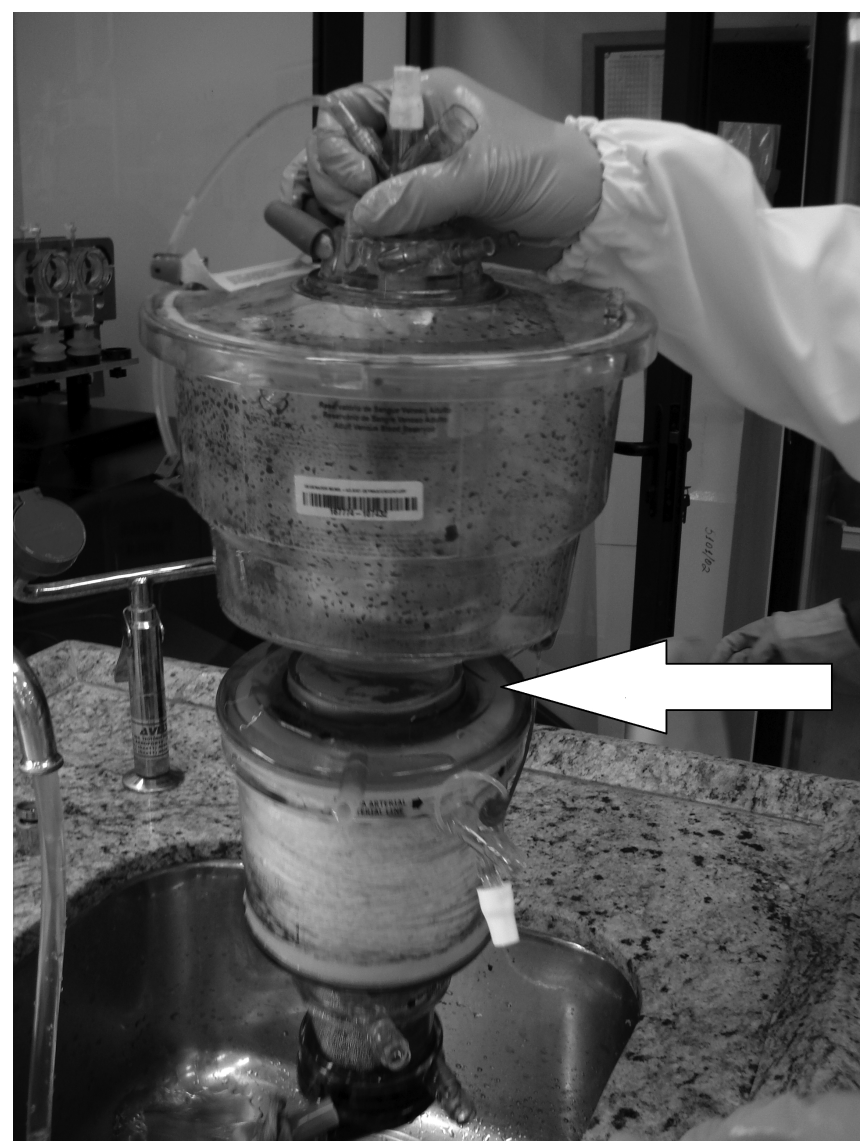

Figure 1 - Oxygenator and Venous Reservoir. The Arrow Indicates the Site of the Crack.
Extracorporeal circulation lasted 35 minutes and the patient was weaned without vasoactive drugs. At the end of the surgical procedure, the patient was transferred to the intensive care unit. After 12 hours, he opened his eyes spontaneously and was extubated 18 hours after the end of the surgery without neurologic sequelae.

The disposable extracorporeal circulation system was secured for technical analysis and the equipment was inspected. Problems were not detected with the ECC equipment; however, the membrane oxygenator had a fissure on its lid that prevented its correct functioning.

Figure 1 reproduces the oxygenator and the assembled venous reservoir. The arrow indicates the crack, reproduced in detail in Figure 2. It shows the difficult visualization of the fissure on the oxygenator lid when the set is assembled. On laboratory testing, when oxygen flow was initiated, movement of the shredded paper placed over the compromised area showed leakage (Figure 3).

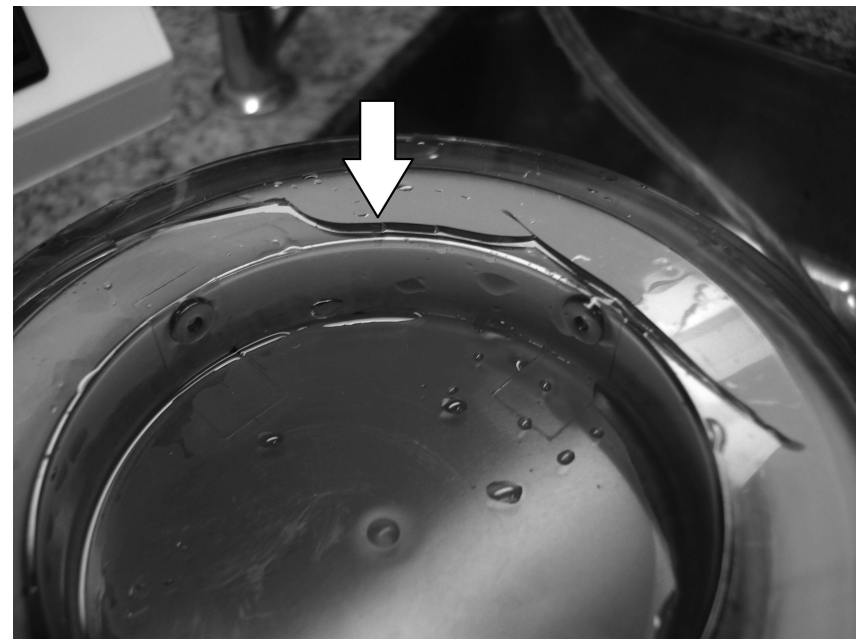

Figure 2 - Detail of the Crack.

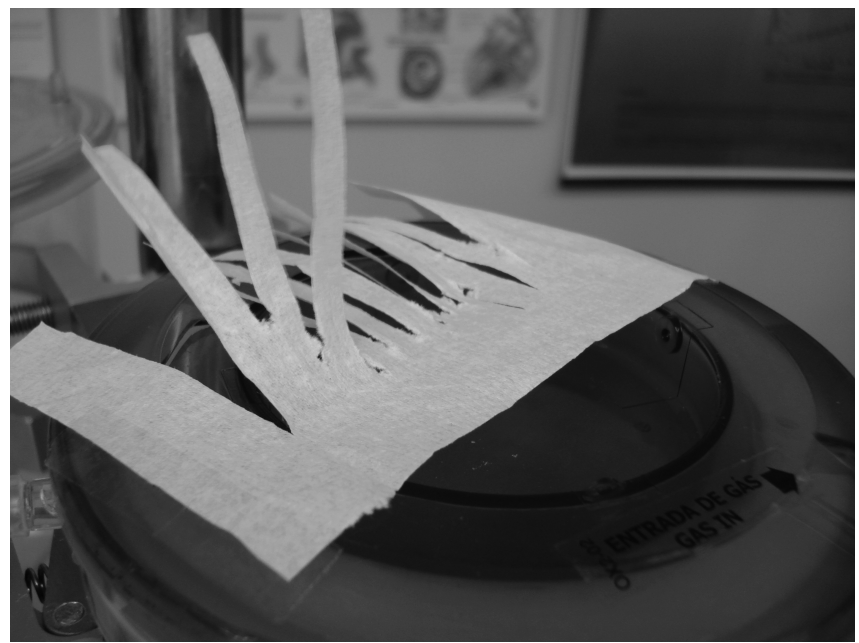

Figure 3 - Paper Movement Indicating Leakage of the Oxygenator. 


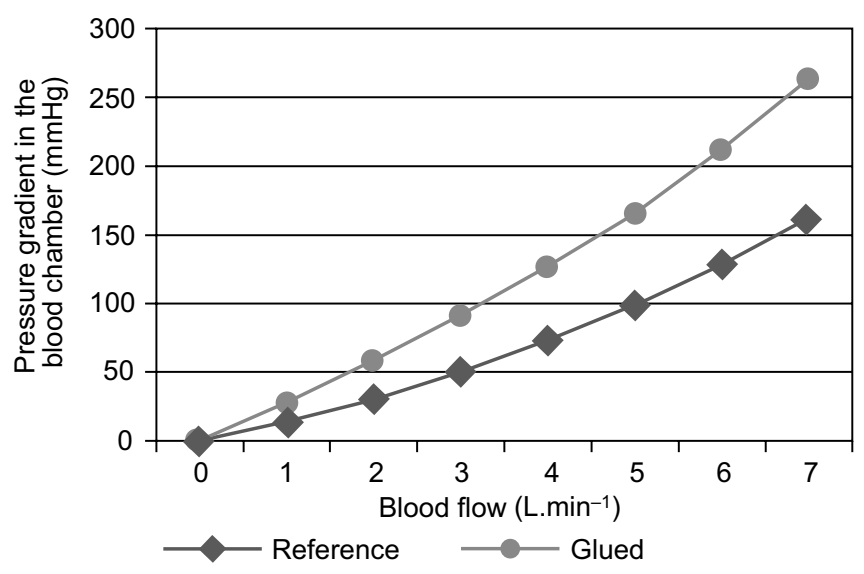

Chart I - Pressure gradient in the blood chamber.

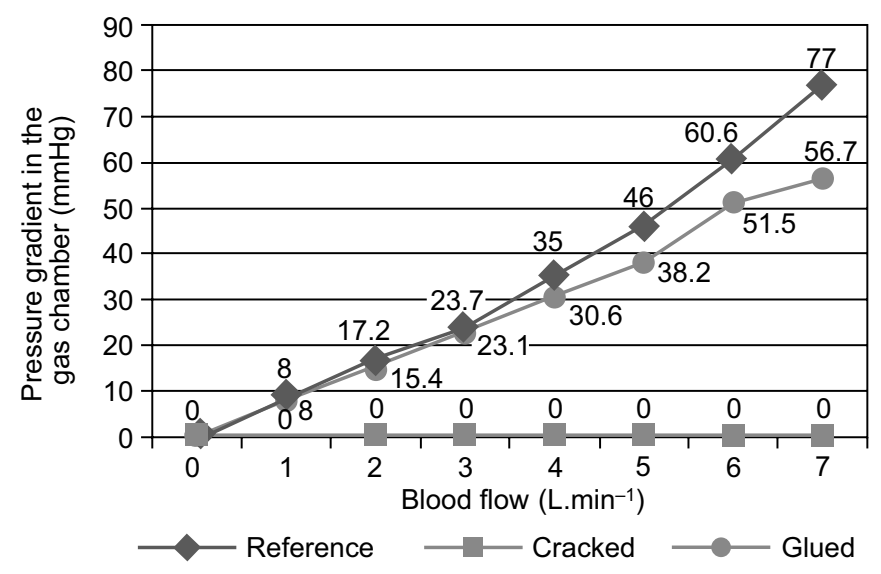

Chart II - Pressure Gradient in the Gas Chamber.

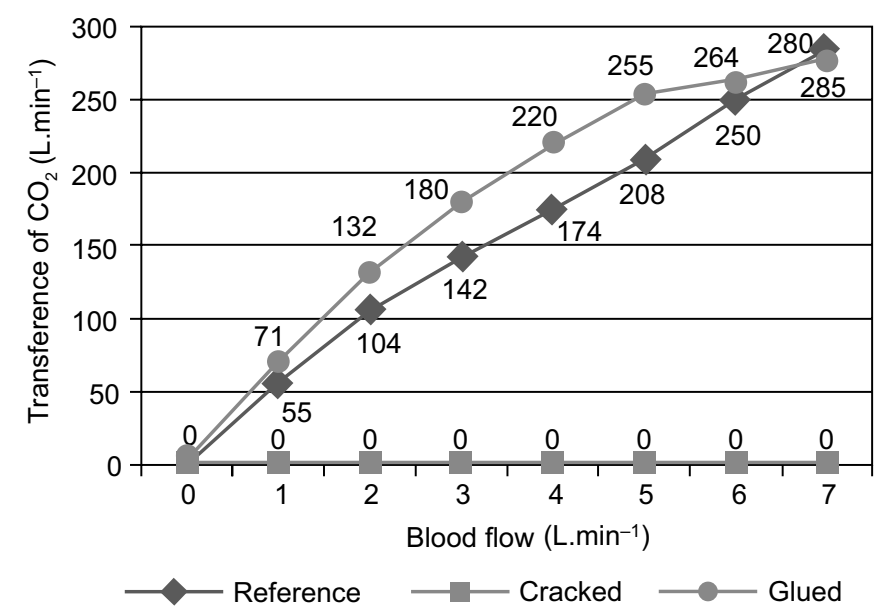

Chart III - Transference of Carbonic Gas.

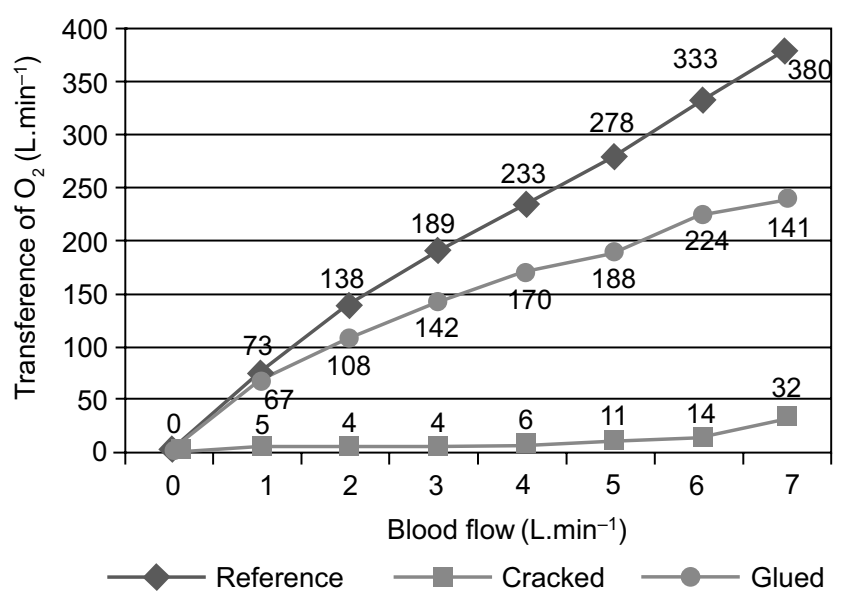

Chart IV - Transference of Oxygen.

The oxygenator underwent gas transference analysis - on a first stage, with the crack and later with the problem corrected (lid repaired with glue). Blood flow for the test was from 1 to 7 L.min-1. The pressure gradient on blood and gas chambers, as well the transference of carbonic gas and oxygen were evaluated. Results are shown in Charts I, II, III, and IV, respectively.

\section{DISCUSSION}

In an American review of critical incidents the authors demonstrated that $82 \%$ of the accidents were related to human errors. The most common errors included disconnection of the respiratory system, exchange of syringes with medications, errors in the control of gas flow, and changes in gas supply. Only $4 \%$ of the incidents with negative outcomes involved equipment failure, attributing great responsibility to human factors ${ }^{5}$.

An Australian study that analyzed 896 incidents reported equipment malfunctioning in 234 cases (26\%). The equipment most commonly involved included infusion pumps and vaporizers. The major contributing factors were failure to check the equipment (37\%), lack of attention (31\%), rush (14\%), and unfamiliar equipment or environment (10\%). The main factors to minimize these errors were rechecking the equipment $(38 \%)$ and detection with the use of monitors (33\%) ${ }^{6}$.

A review of incidents with ECC involving 671,290 procedures over a 2-year period reported 4,882 incidents, and the most common included: reactions to protamine (871), blood dyscrasia (857), water pump failure (371), air or blood clot in the circuit (657), arterial dissection (293), oxygenator failures (272), and mechanical pump failure (260) ${ }^{7}$.

Studies in the United States showed an increased incidence of oxygenator failure. In 1980, 1986, and 1989, the incidence were $1: 56,000,1: 13,000$, and 1:2,700, respectively. Despite this increase in failures, the causes are not always defined ${ }^{8}$. 
The current membrane oxygenators use microporous or silicone-coated polypropylene membranes. They can be classified as plate, coil, or hollow-fiber oxygenators. The latter is used more often and can be subdivided according to what circulates in the interior of the fiber (blood or gas). Circulation of blood inside the fibers can generate an elevated pressure gradient. The flow of gas inside the fiber reduces the trauma produced by the circulation of blood inside capillaries, which allows reducing the required membrane area.

Passage of gas through the membrane depends on its permeability and pressure coefficient between both sides. Permeability is related to the width of the membrane material. Membranes do not have the same permeability to different gases. Most membranes allow passage of carbon dioxide approximately five to six times faster than that of oxygen.

In the case reported here, the crack on the lid of the equipment prevented the correct blood oxygenation. Despite prior verification of the equipment the crack was not seen on the initial inspection. The blue color of the lid and the place where it broke may have contributed for this difficulty.

The absence of a pressure gradient in the gas chamber (Chart II; $p<0.05$ ) even with different blood flow due to the large crack on the oxygenator lid contributed decisively for the low rate of oxygen transference (Chart IV; $p<0.05$ ) and carbonic gas (Chart III; $p<0.05$ ). After repairing the lid of the oxygenator with glue, the pressure gradient in the gas chamber $(p>0.05)$ and consequently the transference of $\mathrm{O}_{2}(p>0.05)$ were closer to the reference value for blood flow below 4 L.min ${ }^{-1}$. Above this flow, a fall in performance was observed $\left(p<0.05\right.$ for $\mathrm{O}_{2}$ transference), which was justifiable since the equipment had already been used and it had areas of coagulated blood that could not be cleared for this test, with consequent increase of the pressure gradient in the blood chamber (Chart l; $\mathrm{p}<0.05$ ). The $\mathrm{CO}_{2}$ transference rate after the lid was fixed surpassed reference values (Chart III; $p<0.05$ ).

In addition to the fracture, the oxygenator and the circuit are subjected to other malfunctions. Gluing failures, and ill fitting connectors or tubings may allow air leakage or entry into the circuit. Connectors whose borders are dented can cause turbulent blood flow with increasing hemolysis. Obstructions due to kinks or angulations can stop blood flow especially in neonatal perfusion in which the size of tubings and cannulas is reduced, requiring relatively elevated flows. This high flow can generate enough resistance in the arterial cannula to push it out of the aorta ${ }^{9}$.

Membrane oxygenators offer resistance to the passage of blood, generating a pressure difference between the equip- ment input and output. Gradient elevation with increased blood resistance can occur due to blood clots on the membrane surface, compromising correct functioning of the equipment and generating high system pressures ${ }^{8}$.

Obstruction to gas escape from the membrane oxygenator can produce air embolism. The venous reservoir should contain a blood volume proportional to the arterial flow to prevent it from emptying and, consequently, a massive injection of air through the arterial pump ${ }^{10}$.

Leakage from the heat-exchanger is another defect difficult to verify. Rupture of weaker points in the exchanger allows transference of water from the heat exchange system to the arterial blood, producing hemolysis, water intoxication, and infection ${ }^{11}$. One should respect the maximum gradient of $10^{\circ} \mathrm{C}$ between the water and arterial blood temperature, especially in cooling and rewarming phases, avoiding micro air embolism secondary to variations in gas solubility at different temperatures ${ }^{12}$.

The observation of a darker shade of red of arterial blood on the exit of the oxygenator helped us with the early detection of hypoxemia. Confirmation was obtained with blood gas analysis. Although the cause of oxygenator failure was not identified, the entire team worked to minimize the damage with palliative measures. Restoration of pulmonary ventilation, temporary bypass between the systemic and pulmonary circulation instituted by the surgeon, and hypothermia were fundamental for the outcome.

There are oximetry and capnography monitoring equipment of ECC gases ${ }^{13,14}$. The analysis of the gas blender and gas expelled from the oxygenator chamber allows monitoring the operation of the blender, analyze the performance of the oxygenation chamber, and measure the carbonic gas output of the oxygenator. The lack of these monitors may have been responsible for the delayed diagnosis.

The role of the perfusionist cannot be underestimated because it is important for the safety of the ECC procedure, either by his/her direct action or that of the equipment. Human errors, lack of preventive maintenance, inadequate use of safety mechanisms, and failure in assembly and verification of equipment represent factors capable of favoring accidents.

Protocols and guidelines have been proposed by services, entities, and organized societies to decrease perioperative incidents.

The interaction among the surgical team, early diagnosis, and immediate intervention were fundamental for the favorable outcome. 